Document downloaded from:

http://hdl.handle.net/10251/62773

This paper must be cited as:

Magraner Rullan, JP.; R. Martinez Val (2014). The birth of airplane stability theory. Proceedings of the Institution of Mechanical Engineers, Part G: Journal of Aerospace Engineering. 228(9):1498-1506. doi:10.1177/0954410013494139.

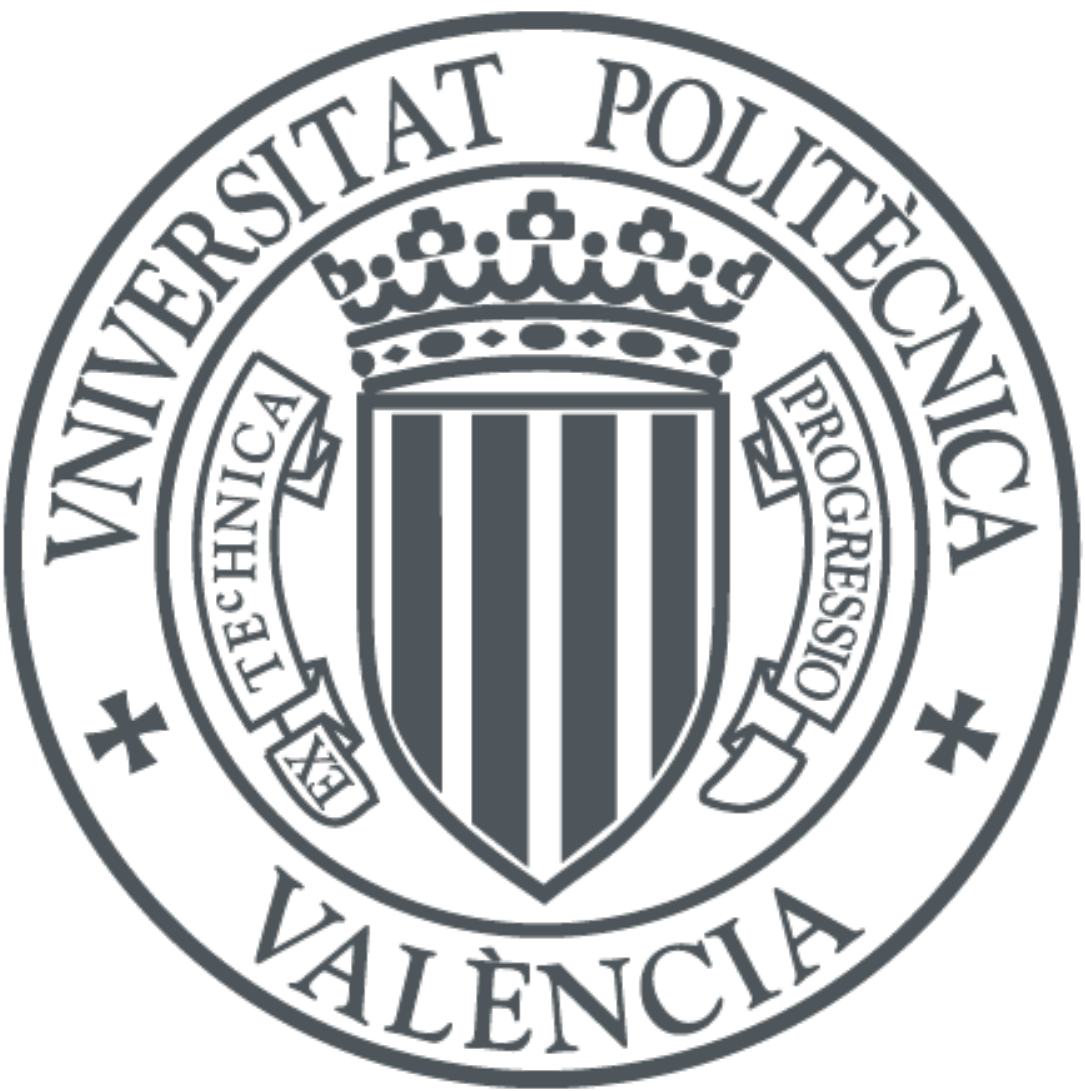

The final publication is available at

http://dx.doi.org/10.1177/0954410013494139

Copyright SAGE Publications (UK and US)

Additional Information 


\title{
The birth of airplane stability theory
}

\author{
J.P. Magraner* and R. Martinez-Val+ \\ *Universidad Politecnica de Valencia, Spain, 46022 \\ +Universidad Politecnica de Madrid, Spain, 28040, corresponding author
}

\begin{abstract}
Airplane stability theory was born at the end of the XIX Century and matured around 100 years ago, when airplanes were hardly controllable yet. The success and safety of flights in the pioneer years depended upon largely unknown stability and control characteristics. Understanding the modes of airplane motion has been of paramount importance for the development of aviation. The contributions made by a few scientists in the decades preceding and following the first flight by the Wright brothers set the concepts and equations that, with minor notation aspects, have remained almost unchanged till present day.
\end{abstract}

Key words: aviation history, airplane stability, phugoid, pioneer Flight Dynamics

\section{INTRODUCTION}

The history of science, from its early times in classical Greece to the XXI Century has witnessed the coexistence of two cultures: on the one hand, a group of educated people who tried to advance knowledge and intellectual capacities; and, on the other hand, the craftsmen who attempted to design and make things without a formal previous idea. In general both cultures evolved apart and had little or no interaction [1].

This vision is also applicable to flight. During the $19^{\text {th }}$ Century the fundamentals of Fluid Mechanics, Thermodynamics, Electricity, Strength of Materials, etc were laid down by numerous scientists: Cauchy, Helmholz, Maxwell, Stokes...; but this was too the era of Cayley, Henson, Stringfellow, Phillips, du Temple, Maxim or Ader [1, 2].

By the time the Wright brothers flew, all the science and mathematics needed to understand and prepare the flight existed in literature [3]. That is why Bryan stated in 1897 [4]: "If any experimenter can so thoroughly master the control of a machine sailing down-hill under gravity as to increase the size of the machine and make it large enough to carry a light motor, and if, further, this motor can be made of sufficient horse-power, combined with lightness, to convert a downward into a horizontal or upward motion, the problem of flight will be solved". Since everything was ready at the turn of century, if the Wright brothers had not performed the first successful flight in December 1903, an airplane would have been flown by someone else sooner or later. As a matter of fact, new designs appeared in the following years: SantosDumont, Curtiss, Bleriot, Latham, Farman ... However, the pioneers faced considerable control troubles since the flying qualities of their aircraft were often less than satisfactory [5, 6]. It was the era of "Those Crazy Young Men and Their Flying Machines" [7].

An excellent survey of Aerodynamics and Control in these early times can be found in Bairstow [8]. Let's mention a few of the main contributors: Bairstow, Bryan, Caldwell, Eiffel, Glauert, Hunsaker, Jones, Lanchester, Prandtl, Riabouchinsky and Von Karman.

Coming back to the XIX Century's visionaries, pioneer glider builders recognized the critical influence of airplane stability. They empirically learned that some degree of stability was essential for the success of their flights [1,9]. Otto Lilienthal, half-way between visionaries and scientists, contributed with numerous aerodynamic measurements that were collected in his book called "Bird flight as the basis of aviation" [10]; he exhaustively reported many manned glider flights, stabilized by small shifts of his own body to properly locate the center 
of gravity; his death put many enthusiasts and inventors (Chanute, Langley, Pilcher, the Wright brothers) in the right path for the first powered flight.

Stability and control come always together, as modern Flight Dynamics teaches [11, 12]. Thus, understanding the modes of motion is of fundamental importance for the flight success and safety. However, the equations governing the airplane motion are so complex that useful exact analytical solutions are not available, even after one century of intensive search [13]. Flight Dynamics requires concepts and tools from Mathematics, Mechanics, Aerodynamics, instrumentation, simulation, pilot handling, ergonomics, etc [14]. This multidisciplinarity emphasizes the crucial value of the first contributors in the decades just preceding and following the first flight.

The next chapter presents an overview of the pioneer stability theories, namely those developed by Zhukowsky, Bryan and Williams, Lanchester, Painlevé and Bothezat. For various reasons their models were incomplete, included errors or hardly reached the Flight Dynamics community. Then, it was Bryan, in 1911, with his book called "Stability in Aviation" [15], who created the definitive basis of our current Flight Dynamics. As it will be shown in the third section of this paper, except for minor notation aspects, his equations have remained unchanged and perfectly useful for aerospace scientific and engineering purposes.

\section{PIONEERS OF AIRCRAFT STABILITY}

The mathematical language, with its concepts and tools, has been developed earlier than the physical theories it serves. That may explain why many great scientists were, actually, applied mathematicians who efficiently contributed to the advancement of Science. As it will be shown in the next paragraphs this was also the case with pioneer theories of airplane stability. Among the contributions made by scientists and engineers in the decades just preceding and following the first successful powered flight, this section will be focused on six works that were particularly remarkable, for its intrinsic value or its influence in later developments of Flight Dynamics: Zhukowsky (twice), Bryan and Williams, Lanchester, Painlevé and Bothezat. The definitive book by Bryan in 1911 will be more extensively described in Section 3. Only key ideas will be presented, slightly modified in nomenclature to facilitate its understanding. Other coetaneous works by Zahm, Penaud, Crocco, etc will not be considered here, but the interested reader can find detailed historical overviews in refs 5 and 7.

\subsection{Zhukowsky (1891)}

In 1891, the Russian physicists Nicolai Zhukowsky published his first paper related to flight with the suggesting tittle of "On soaring of birds" [16]. The paper was not a mere phenomenological description of bird flight, but a mathematical analysis of acting forces and resulting motion.

Zhukowsky idealized the wing as a flat plate and studied the center of gravity motion under the effect of weight and the aerodynamic forces, acting along and perpendicular to the trajectory, as shown in Fig.1.

The dynamic equilibrium along and normal to the trajectory provides

$$
\begin{aligned}
& d\left(\frac{m V^{2}}{2}\right)=m g d z-F d s \\
& N+m \frac{V^{2}}{r}=m g \cos \gamma
\end{aligned}
$$


Where $F$ and $N$ are the components of the aerodynamic forces (drag and lift in today's nomenclature), $m$ is mass, $V$ speed, $d s$ the differential element along the trajectory, $r$ its radius of curvature and $\gamma$ its glide slope.

The study included the hypotheses of small, constant angle of attack $i$, negligible effect of the aerodynamic drag, $F$, and initial height and speed $h_{0}$ and $V_{0}$, respectively. These hypotheses allowed rearranging Eqs 1 and 2 as

$$
\begin{aligned}
& V^{2}-V_{0}^{2}=2 g\left(z-h_{0}\right) \\
& A+\sin \gamma \frac{d \gamma}{d z}=\frac{g \cos \gamma}{V^{2}}
\end{aligned}
$$

where

$$
A=\frac{0.13 S \sin i \cos i}{m}
$$

finally leading to a unique differential equation

$$
A+\sin \gamma \frac{d \gamma}{d z}=\frac{g \cos \gamma}{2 g\left(z+h_{0}\right)}
$$

that was integrated as

$\cos \gamma=\frac{2 A}{3}\left(z+h_{0}\right)+\frac{\sqrt{h_{0}}\left(\cos \gamma_{0}-\frac{2 A h_{0}}{3}\right)}{\sqrt{z+h_{0}}}$

The same equation was later published by Lanchester in his "Aerodonetics" [17].

It must be noticed that since Zhukowsky was mainly interested in the center of gravity motion he did not included any equation on pitching. Depending upon the values of parameter $A, \mathrm{Eq}$ 6 represents a variety of solutions, as depicted in Fig. 2. In particular, the semicircular arcs of trajectory E correspond to the stability limit. In spite of its intrinsic relationship to flight stability, Zhukowsky did not mention such circumstances in his paper.

\subsection{Bryan and Williams (1904)}

Professor George Bryan was an eminent applied mathematician, highly interested in physics $[6,7]$. After an earlier career on Thermodynamics [18], he discovered manned gliders thanks to his friend Percy Pilcher, one of Lilienthal's disciples, and devoted part of his working capability to the analysis of natural and artificial flight $[4,18]$. The tragic death of his friend, Pilcher, three years after that of his German mentor, prompted Bryan into the study of flight stability and safety. Then, with one of his students, Williams, published a fairly complete analysis entitled "The longitudinal stability of aerial flights" [19].

As in Zhukowsky's analysis, Bryan and Williams considered the glide of a flat plate, but they introduced a third equation to account for the pitching motion of the plate around its center of gravity. Following Prof Routh's nomenclature [20] they wrote the flight dynamic equations as 


$$
\begin{aligned}
& m\left(\frac{d u}{d t}+q \cdot w-r \cdot v\right)=-X-m g \sin \theta \\
& m\left(\frac{d w}{d t}+p \cdot v-q \cdot u\right)=-Z+m g \cos \theta \cos \phi \\
& I_{y y} \dot{q}+I_{x z}\left(p^{2}-r^{2}\right)+\left(I_{x x}-I_{z z}\right) p r=-M
\end{aligned}
$$

where $m$ is mass, $u, v$ and $w$ the horizontal longitudinal, traverse and vertical speed components, respectively, and $p, q$ and $r$ the angular velocities with respect to the three coordinate axes.

The main contribution of Bryan and Williams was the study of small oscillations around a generic equilibrium, of steady motion, following the classical linear expansion of the acting aerodynamic forces. After rearranging the equations they arrived at the stability matrix

$$
[S M]=\left(\begin{array}{ccc}
\left(\frac{X_{u}}{m}+\lambda\right) & \frac{X_{w}}{m} & \left(\lambda \frac{X_{q}}{m}-g \cos \theta_{0}\right) \\
\frac{Z_{u}}{m} & \left(\frac{Z_{w}}{m}+\lambda\right) & \lambda\left(U_{0}+\frac{Z_{q}}{m}\right)+g \sin \theta_{0} \\
\frac{M_{u}}{I_{y}} & \frac{M_{w}}{I_{y}} & \left(\lambda \frac{M_{q}}{I_{y}}+\lambda^{2}\right)
\end{array}\right)
$$

whose determinant equaled to zero provided

$$
\operatorname{det}[S M]=\lambda^{4}+B \lambda^{3}+C \lambda^{2}+D \lambda+E=0
$$

To ensure that the oscillations are stable, $B, C, D$, and $E$ must be positive and the parameter $D(B C-D)-B E^{2}$ must also be positive [21].

Bryan and Williams used Langley's expressions [22] for the relationship between aerodynamic force, $R$, and speed, $V$, as

$$
R=K S V^{2} f(\alpha)
$$

$S$ being the flat plate area and $\alpha$ the angle of attack. For small angles of attack $f(\alpha) \approx \sin \alpha$.

The center of pressure is assumed to be located, as suggested by Joessel [23].

$$
x_{c p}=\frac{c}{2} \phi(\alpha)
$$

with

$$
\phi(\alpha)=0.6(1-\sin \alpha)
$$


Since the only force interaction between air and plate is due to pressure, the resulting aerodynamic force is perpendicular to that one.

The main conclusions drawn from ref.19 are:

- Small oscillations of a gliding flight are determined by the roots of a quartic equation and the conditions prescribed by Routh;

- The coefficients of the quartic equation depend on the physical features of the glider and on the partial derivatives of the aerodynamic forces and moments with respect to the horizontal and vertical speeds and to the angular pitching speed;

- When the glider is composed of a number of wings, the various contributions can be considered separately and then added to find the overall forces and moments, and the location of the center of pressure;

- The longitudinal stability of gliders can be investigated mathematically. The method shown should be applied to any new flying machine before attempting actual flights; and

- The conditions for stable straight glides can be represented as $V^{2}>K a$, where $a$ depends on the linear dimensions of the glider and $K$ on the aspect ratio, glide angle and law of aerodynamic force.

Interestingly, the first four conclusions are still valid more than a hundred years after they were written [8].

\subsection{Zhukowsky (1906)}

Fifteen years after his first paper on stability, Zhukowsky published a second work with important contributions to both Aerodynamics and Stability. He analyzed the motion of a rectangular flat plate, falling while rotating around an axis parallel to its longer side in calm air, idealizing the flow as indicated in Fig. 3 [24]. Due to the combined effect of glide and rotation, the streamlines surrounding the plate close at a point downstream, somehow like an airfoil. He provided a crucial link between aerodynamic lift and plate rotation in the wellknown form of

$$
\frac{L}{b}=\rho V \Gamma
$$

where $L$ is lift, $b$ the plate span, $\rho$ air density, $V$ speed and gamma the circulation due to the rotation effect.

Zhukowsky assumed that the plate center of gravity followed an almost straight trajectory as:

$$
\begin{aligned}
& \frac{m V^{2}}{r}=m g \cos \gamma-L \\
& m \frac{d\left(V^{2} / 2\right)}{d x}=m g \sin \gamma-D
\end{aligned}
$$

In steady motion

$$
\frac{m V^{2}}{r=\infty}=0=m g \cos \gamma_{0}-L_{0}
$$


$m \frac{d V^{2}}{2 d x}=0=m g \sin \gamma_{0}-D_{0}$

To analyze the stability of such a motion, he introduced two approximations:

$\frac{1}{r}=\frac{\frac{d^{2} y}{d x^{2}}}{\left(1+\left(\frac{d y}{d x}\right)^{2}\right)^{\frac{3}{2}}}=\frac{d^{2} y}{d x^{2}}$

$$
\Delta \gamma \approx \frac{d y}{d x}
$$

and rearranged the perturbed form of Eqs. 16 and 17 into

$$
\frac{d^{3} y}{d x^{3}}+\left(\frac{3 g \sin \gamma_{0}}{V_{0}^{2}}\right) \frac{d^{2} y}{d x^{2}}+\left(\frac{2 g^{2}}{V_{0}^{4}}\right) \frac{d y}{d x}=0
$$

leading to

$$
\frac{d y}{d x}=C_{1} e^{\lambda_{1} x}+C_{2} e^{\lambda_{2} x}
$$

which results in

$$
\lambda_{1,2}=\frac{g}{V_{0}^{2}}\left(-\frac{3}{2} \sin \gamma_{0} \pm \sqrt{\frac{9}{4} \sin ^{2} \gamma_{0}-2}\right)
$$

Since the natural motion is a descending glide, the motion is always stable. With the current nomenclature and a parabolic wing drag polar we could write

$$
\lambda_{1,2}=\left(-\frac{3}{4} \frac{\rho S}{m} C_{D} \pm i \frac{g \sqrt{2}}{V_{0}^{2}}\right)
$$

The period of oscillation would be

$$
T_{2 \pi}=\pi \sqrt{2} \frac{V_{0}}{g}
$$

Which is the value given by Lanchester for the phugoid oscillation in "Aerodonetics" [17]. Equation 25 also shows the damping effect of the aerodynamic drag. Although these last results did not explicitly appear in the paper, they constitute a proof of the high value of Zhukowsky's contributions. 


\subsection{Lanchester (1908)}

Frederick W. Lanchester was not actually a scientist, but a mechanical engineer with a great enthusiasm for mechanical devices. He devoted most of his efforts to the construction of innovative motor cars, but also oriented his inventive mind towards free-flying gliders [9]. $\mathrm{He}$ investigated too into practical Aerodynamics and speculated over the relationship between the vortex wake and the drag due to lift, but was not able to set up a suitable theory to quantify that relationship. He largely debated with Prandtl over the authorship of the induced drag dependence $[25,26]$.

However, his main contributions were in the field of flying machine stability. Lanchester published detailed accounts of his flying experiments in "Aerodonetics" [17] and wrote a companion book on "Aerodynamics" [27]. Lanchester's pine wood hand gliders were inherently stable and exhibited an undulating path that he named "phugoid". He noted that if the glider was launched at a certain "natural speed" it would glide as if on rails, but in general the glider followed an oscillatory motion as depicted in Fig. 4 [7]. He deduced that this behavior involved an interchange between kinetic and potential energies.

The glider stability analysis was based on four hypotheses: 1) the flight is symmetric, in a vertical plane, with three degrees of freedom (two linear coordinates on the plane and the pitching angle); 2) the motion is conservative (i.e. either there is no drag or it is permanently compensated by thrust); 3 ) there is no inertia moment with respect to the pitching motion; and 4) the size of the glider is much smaller than the radius of curvature of the trajectory. Lanchester arrived at the same differential equation proposed by Zhukowsky (Eq. 6) but with his own nomenclature as

$$
\frac{d(\cos \theta(H))}{d H}=\frac{\cos \theta(H)}{2 H}-n
$$

that can be integrated to

$$
\cos \theta(H)=\frac{2}{3} n H+\frac{C}{\sqrt{H}}
$$

The particular case when $C=0$ corresponds to semicircular trajectories, numbered 4 in the plot of Fig. 5. Smaller oscillations are naturally damped. However, for very large oscillations the path is completely perturbed, denoting that the stability limits have been exceeded [17].

Although, as indicated earlier, Zhukowsky had implicitly obtained some of the main features of the phugoid mode, it was Lanchester in 1908 who published the first approximation of the phugoid frequency [28] as

$\omega_{p}=g \frac{\sqrt{2}}{V}$

Lanchester was aware of the limitations of his theory and identified some improvements to achieve more realistic results. Thus, the aerodynamic drag produces a damping effect whilst the inertia moment amplifies the natural perturbations. The corrections had phenomenological rather than mathematical basis.

The beauty of Lanchester's work, and the origin of his enduring success, was its practicality and its theoretical simplicity [6]. 
Paul Painlevé, a French mathematician, was Professor of Applied Mechanics and Machines at École Polytechnique during the pioneer years of aviation. Most of his works were devoted to Algebra and Differential Equations but, as many educated French men of this time, he became enthusiast of balloons and gliders, due to the ambiance created by scientific journals and popular technical magazines. During the first decade of the XX century he worked on the fluid mechanics and stability of flight [29-31]. Of particular interest for this historical review is the "Theory of airplanes", included in his course on Mechanics and Machines [29]. Interestingly, after the First World War he was deeply involved in politics and became Minister and Primer minister several times.

On its side, Georges de Bothezat, a Russian engineer, went to Paris for his Doctorate studies and elaborated a Thesis on airplane stability [32], under the guidance of Paul Painlevé. He later returned to Russia, but fled to the United States after the Revolution, to continue his research on propellers and helicopters, finally becoming a businessman [33-36].

Coming back to Painlevé, he analyzed the glide of a lifting plate, as sketched in Fig. 6. He assumed Joessel's hypothesis [23] for the centre of pressure, and stated that the stability of the gliding motion required the decline of all transients. Let's be $\mathrm{F}$ the aerodynamic drag and $\mathrm{N}$ the aerodynamic force normal to speed, formulated according to Eiffel's expressions [37, 38].

$F=-V^{2}\left(\lambda \alpha^{2}+\mu\right)$

$N=\lambda V^{2} \alpha$

In the former equations $\lambda$ and $\mu$ are constant coefficients. The aerodynamic pitching moment is expressed as

$v=\lambda \sigma V^{2}\left(\alpha-\alpha_{1}\right)$

where $\alpha_{l}$ is the "right" (i.e. equilibrium) angle of attack and $\sigma$ a constant, positive if the airplane is stable.

The centre of gravity and pitching motions obey the intrinsic equations

$M \frac{d V}{d t}=-V^{2}\left(\lambda \alpha^{2}+\mu\right)-P \gamma$

$M V \frac{d \gamma}{d t} \approx-\lambda V^{2} \alpha-P$

$I_{y} \frac{d^{2} \theta}{d t^{2}}=\lambda \sigma V^{2}\left(\alpha-\alpha_{1}\right)-2 h V \frac{d \theta}{d t}$

The term $2 h V d \theta / d t$, in Eq. 35 introduces certain pitching damping. If Equations 33 and 34 are linearized around an equilibrium state, the longitudinal and normal equations, with the current definition of $C_{L}$ and $C_{D}$, become

$$
\begin{aligned}
& \frac{d V}{d t}=-\frac{\rho S V_{0}^{2}}{2 m} \Delta C_{D}-\frac{\rho S C_{D} V_{0}}{m} \Delta V-g \cos \gamma_{0} \Delta \gamma \\
& \frac{d \gamma}{d t}=\frac{\rho S V_{0}}{2 m} \Delta C_{L}+\frac{\rho S C_{L} V_{0}}{m} \Delta V+\frac{g \sin \gamma_{0}}{V_{0}} \Delta \gamma
\end{aligned}
$$

Painlevé's solutions corresponding to constant glide angle $(d \gamma / d t=0)$ provide

$$
\frac{d V}{d t}=-\frac{\rho S V_{0} C_{D}^{2}}{m} \frac{\partial}{\partial C_{L}}\left(\frac{C_{L}}{C_{D}}\right)
$$

This equation, known as "Painlevé's theorem", indicates that the motion is stable in speed if the airplane flies at small angles of attack, but unstable at high angles of attack.

Bothezat worked further on Painlevé's equations, with a six degrees of freedom motion model, completely independent from that one studied by Bryan [15] which appeared several months later than Bothezat's Thesis. 
Figure 7 shows the airplane used for the stability studies, consisting of a fixed horizontal stabiliser at the rear, the wing and a separated, all moving elevator at the front of the aircraft. His longitudinal stability findings are very complete and contradictory to previous hypotheses and results. For example, Bothezat states that the airplane longitudinal stability needs not any particular hypothesis on the centre of pressure location, such as the Joessel's model: it is enough that the horizontal tail incidence is smaller than that of the wing. The results include diverse types of perturbation decay as shown in Fig. 8. Although he studied the lateraldirectional stability too, in the last chapter, the scope and depth of this section is of lesser interest.

\section{BRYAN'S DYNAMIC AIRPLANE STABILITY}

As stated above, Prof. Bryan was a brilliant applied mathematician who became enthusiast of flight and its problems [6, 7] and, eventually, after the death of his friend Pilcher, decided to aim his efforts towards airplane stability and safety. The interested reader can find a highly detailed account of his prolific life and contributions in ref. 18.

When Prof Bryan wrote his 1904 paper on the stability of aerial flights [19] the Wright brothers had just performed their first set of powered flights, but this crucial event was kept hidden. However, within a few years a number of intrepid flyers (Santos-Dumont, Farman, Curtiss, Bleriot, Graham White, Fernandez and many others) were able to fly. The Wright brothers traveled to Europe to counterbalance the growing activity and to show that they were well ahead other designers. Albeit most machines were hardly controllable, Bleriot was capable of crossing the English Channel on 25 July 1909 and many inventors participated with great expectation and success in the Reims Aviation Week one month later.

During these years Zhukowsky, Lanchester and others put some pieces of the stability puzzle, but Prof Bryan realized that it was far from complete and took the initiative of elaborating a definitive and more rigorous treatise on airplane stability [39].

Bryan adopted the modern concept of stability, unveiled a few decades earlier: "a dynamical system is said to be stable or to possess stability if, when slightly disturbed from a state of equilibrium, it tends to return to and remain in that state, the disturbance acting only for a finite time" [5].

\subsection{Equations and modes}

According to Bryan, the motion of an aircraft and its stability and control could be determined completely by dynamical principles. His formulation was based on two key assumptions [40]: first, the aerodynamic forces and moments depend only on instantaneous values of the motion variables; and, second, the aerodynamic forces and moments could be linearized with respect to the motion variables.

Following the rigid body dynamics, the airplane motion is studied through six equations, corresponding to the six degrees of freedom: three linear space coordinates, plus three angular coordinates.

With the assumption of both inertial and aerodynamic symmetry, and the aircraft being subjected only to symmetric disturbances, the lateral asymmetric motion can be separated from the longitudinal motion if the asymmetric perturbations are small. In other words, once the equations are linearized, the airplane motion can be decomposed into the longitudinal and lateral-directional modes [7]. Only the longitudinal stability will be considered here.

Bryan introduced the concept of "rotative" derivative, to mean partial derivative with respect to the pitching angular velocity 
$f_{q}\left(\alpha_{0}\right)=\left.U_{0} \frac{\partial f(\alpha)}{\partial q}\right|_{\alpha_{0}}$

$\phi_{q}\left(\alpha_{0}\right)=\left.U_{0} \frac{\partial \phi(\alpha)}{\partial q}\right|_{\alpha_{0}}$

Since Bryan followed Joessel's aerodynamic formulations (see Eqs. 13 and 14), he arrived at the conclusion that the glide of a simple flat plate could only be stable if the center of pressure shifted rearwards on increasing the angle of attack.

But airplanes were not conceived as wing-alone aircraft. Instead, all airplanes had a horizontal stabilizer as shown in Fig. 9, modified from ref. 15. To avoid any pitching moment in the equilibrium flight, the center of pressure must coincide with the center of gravity. Bryan knew the downwash effect on the rear plane but did not take it into account to avoid entangling the formulas. Another limitation of Bryan's theory is that the lift curve slope of wing and tailplane are equal, which overestimate his stability results.

The longitudinal stability quartic was simplified by Bryan in several steps that provided interesting physical insight into the oscillation modes. For example, to ensure airplane stability, the distance from wing to tailplane, $l_{t}$, should be

$l_{t}>\frac{K}{m} \frac{S_{1}\left(S_{1}+S_{2}\right)}{S_{2}} k^{2}$

where $K$ is the constant appearing in the aerodynamic force formula (Eq. 12) and $k$ the y-axis inertia moment radius.

Bryan realized that there were two different modes: the "slow" or "long oscillation mode" and the "short oscillation mode". For the long oscillation mode (equivalent to Lanchester's phugoid) the imaginary part of the root is, in first approximation,

$$
\lambda_{B}= \pm i \sqrt{\frac{E_{0}}{C_{0}}}
$$

$E$ and $C$ being the independent and $\lambda^{2}$ coefficients of Eq. 11, respectively. This result easily becomes

$$
T_{B}=2 \pi \sqrt{\frac{C_{0}}{E_{0}}}
$$

that can be converted into

$$
\lambda_{B}= \pm i \sqrt{2} \frac{g}{U_{0}}
$$

This last result was not published by Bryan, although it was implicit in his analysis.

Regarding the damping of the long oscillation, Bryan obtained the following expression 
$\left(\frac{B_{0} E_{0}-C_{0} D_{0}}{2 C_{0}{ }^{2}}\right) \approx-\frac{R_{0}{ }^{*}}{2 C_{0}{ }^{2}}$

which, with current nomenclature of aerodynamic drag, can be expressed as

$-\frac{R_{0}^{*}}{2 C_{0}^{2}} \approx-\frac{\rho S_{1} U_{0} C_{D}}{2 m}$

The former equations constitute just an example of Bryan's analysis of airplane stability. $\mathrm{He}$ had the vision on how to study the problem and how to solve control troubles in new designs. Albeit the book appeared one hundred years ago, the notation used is perfectly understandable nowadays [5]. Bryan emphasized that all approximations to the longitudinal modes should satisfy the fourth-order longitudinal characteristic polynomial, to be both reliable and useful [41].

\section{CONCLUDING REMARKS}

Flight Dynamics has played a crucial role in the continuing development of the frontiers of aerospace. Knowing the preliminary stability concepts is important to understand the current status of the discipline. The weak and strong points of their contributions may provide some clues to foster science and technology a step forward.

Although better known for his works on Aerodynamics, Zhukowsky was the first to elaborate a mathematically sound study on the stability of glider flight. His equations showed in 1891 the oscillatory nature of the longitudinal motion and, in 1906, provided an implicit value for the phugoid frequency. However, he was not aware of the importance of his findings and stopped the analysis just before realizing and discussing the stability limits. His works were not fully appreciated until the 20s, when the contributions by Lanchester and Bryan had already been widely disseminated. Similarly, Painlevé's and Bothezat's works on airplane stability were almost unknown by the Flight Dynamics community in early times, probably because they were written in French but for other reasons too. Painlevé left Academia and is much more known for his political career. On another side, Bothezat had to stop his promising research in Russia, emigrate to USA and devote his capabilities to new undertakings. Although he was the first to conceive and solve the six-degrees of freedom model of airplane motion, his name is not duly recognised among the stability pioneers.

Conversely, Lanchester and Bryan are common references in current research work and text books [3, 5-9, 14, 28, 39-44]. Lanchester, mainly for his early description of the phugoid mode, soon accepted and widely known through literature. Bryan, for developing the general equations of airplane motion, applied to a six degrees of freedom aircraft, thus providing a solid mathematical basis for two and three-dimensional analysis of flight. He did not only discussed and improved Lanchester's analysis of the slow oscillatory phugoid motion, but unveiled the existence of a short period mode. Because of his papers and his seminal book, he is recognized as the real originator of Flight Dynamics [5-9]. Bryan's equations, with very few changes, are still in everyday use and are the basis of closed-loop control applications as well as of the design of flight simulators, among other recent developments [9].

Bryan's "Stability in Aviation" [15] and Lanchester's "Aerodonetics" [17] are examples of that small category of books that continue to be widely referenced and in their plenitude, although rarely read, a hundred years after its publication [18]. 


\section{ACKNOWLEDGEMENTS}

The authors appreciate the valuable help of Artem Dombrovsky, former student at Universidad Politecnica de Valencia, for his translation of the papers by Nikolai Zhukowsky that were written in Russian.

\section{REFERENCES}

[1] Anderson, J.D. The Airplane: A History of its Technology, AIAA, Reston, VA. 2002.

[2] Moolman, V. The road to Kitty Hawk, Time Life, Amsterdam, The Netherlands, 1980.

[3] Etkin, B. Dynamics of Atmospheric Flight, John Wiley and Sons, New York, NY, 1972.

[4] Bryan, G.H. Review of artificial flight, Science Progress, London, United Kingdom, 1897.

[5] Babister, A.W. Aircraft Stability and Control, Pergamon Press, New York, NY, 1961.

[6] Cook, M.V. Flight Dynamics Principles, $2^{\text {nd }}$ Edition, Elsevier, Amsterdam, The

Netherlands, 2007.

[7] Perkins, C.D. Development of Airplane Stability and Control Technology. Journal of Aircraft, Vol. 7, No 4, 1970, pp. 290-301.

[8] Bairstow, L. Aerodinamica aplicada (in Spanish), INTA, Madrid, Spain, 1946.

[9] Abzug, M.J. and Larrabee, E.E. Airplane Stability and Control, $2^{\text {nd }}$ Edition, Cambridge

University Press, Cambridge, United Kingdom, 2002.

[10] Lilienthal, O. Der Vogelflug als Grundlage der Fliegekunst (in German), R. Gartner, Berlin, Germany, 1889.

[11] Torenbeek, E. and Wittenberg, H. Flight Physics. Essentials of Aeronautical Disciplines and Technology with Historical Notes, Springer, Dordrecht, The Netherlands, 2009.

[12] Anderson, J.D. Introduction to Flight, $6^{\text {th }}$ Edition, McGraw-Hill, New York, NY, 2008.

[13] Graham, W.R. Asymptotic analysis of the classical aircraft stability equations. The Aeronautical Journal, Vol. 103, February 1999, pp. 95-103.

[14] Hancock, G.J. An Introduction to the Flight Dynamics of Rigid Aeroplanes, Ellis Horwood, New York, NY, 1995.

[15] Bryan, G.H. Stability in Aviation, MacMillan, London, United Kingdom, 1911.

[16] Zhukowsky, N.E. On soaring of birds (in Russian), Mathematical Society of Moscow, 1891, in Collected Papers, Vol. V, pp. 7-35, edited by Prof. A. P. Kotelnikov, People`s Commissariat of Heavy Industry of the URSS, Moscow, Russia, 1937.

[17] Lanchester, F.W. Aerial Flight: Part 2, Aerodonetics, Constable, London, United Kingdom, 1908.

[18] Boyd, T.J.M. One Hundred Years of G.H. Bryan's Stability in Aviation. Journal of Aeronautical History, Paper No 2011/4, 2011, pp. 97-115.

[19] Bryan, G.H. and Williams, W.E. The longitudinal stability of gliders. Proc. Royal Society, Vol. A73, 1904, pp. 100-116.

[20] Routh, E.J. Advanced Rigid Dynamics, MacMillan, London, United Kingdom, 1879.

[21] Routh, E.J. A Treatise on the Stability of a given state of motion, particularly steady motion, MacMillan, London, United Kingdom, 1877.

[22] Langley, S.P. Experiments in Aerodynamics, Smithsonian Institution, Washington, DC, 1981.

[23] Joessel, J. Rapport sur des expériences relatives aux gouvernails. Mémorial du Génie Maritime, Rapport 9, 1873.

[24] Zhukowsky, N.E. De la chute dans l'air de corps légers de forme allongèe, animés d'un mouvement rotatoire (in French), Bulletin de l'Institut Aérodynamique de Koutchino, Fasc. 1, 1906, in Collected Papers, Vol. V, pp. 82-98, edited by Prof. A. P. Kotelnikov, People`s Commissariat of Heavy Industry of the URSS, Moscow, Russia, 1937. 
[25] Von Karman, T. Aerodinámica: temas seleccionados a la luz de su desarrollo histórico, (in Spanish), INTA, Madrid, Spain, 1954.

[26] Wegener, P.P. What makes airplanes fly? History, science and applications of aerodynamics, 2nd Edition, Springer, New York, NY, 1997.

[27] Lanchester, F.W. Aerial Flight: Part1, Aerodynamics, Constable, London, United

Kingdom, 1907.

[28] Pradeep, S. and Kamesh, S. Does the Phugoid Frequency Depend on Speed? Journal of Guidance Dynamics and Control, Vol. 22, N², 1999, pp. 372-373.

[29] Painlevé, P. Cours de Mécanique et Machines (in French). École Polytechnique, Paris, France, 1909.

[30] Painlevé, P. and Borel, E. L'Aviation (in French). Felix Alcan, Paris, France, 1911.

[31] Beauplan, R. Nécrologie de Paul Painlevé (in French). L'Illustration n 4731, 4 novembre 1933

[32] Bothezat, G. Étude de la Stabilité de l'Aéroplane (in French). Thése présentée à la Faculté des Sciences de Paris, France, Janvier 1911.

[33] Bothezat, G. The General Theory of Blade Screws. NACA Report n 29, 1920.

[34] Bothezat, G. General Theory of the Steady Motion of an Airplane. NACA Report n ${ }^{\circ} 97$, 1921.

[35] Boulet, J. L'histoire de l'hélicoptère: racontée par ses pionniers 1907-1956 (in French). France-Empire, Paris, France, 1991.

[36] Allen, P. The helicopter: an illustrated history of rotary-winged aircraft. Airlife, Shrewsbury, United Kingdom, 1996.

[37] Eiffel, G. Recherches expérimentales sur la résistance de l'air. Mémoires de la Société des ingénieurs civiles de France, Bulletin 1907.

[38] Eiffel, G. Installation d'un laboratoire d'aérodynamique. Mémoires de la Société des ingénieurs civiles de France, Bulletin janvier 1910.

[39] Phillips, W.F. Phugoid Approximation for Conventional Airplanes. Journal of Aircraft, Vol. 37, N 1, 2000, pp. 30-36.

[40] Pamadi, B.N. Performance, Stability, Dynamics and Control of Airplanes, $2^{\text {nd }}$ Edition, AIAA, Reston, VA, 2004.

[41] Ananthkrishnan, N. and Ramadevi, P. Consistent Approximations to Aircraft

Longitudinal Modes. Journal of Guidance Dynamics and Control, Vol. 25, No 4, 2002, pp. 820-824.

[42] McRuer, D., Ashkenas, I. and Graham, D. Aircraft Dynamics and Automatic Control, Princeton University Press, Princeton, NJ, 1990.

[43] Russell, J.B. Performance and Stability of Aircraft, Arnold, London, United Kingdom, 1996.

[44] Phillips, W.F. Mechanics of Flight, $2^{\text {nd }}$ Edition, John Wiley and Sons, Hoboken, NJ, 2010 .

\section{FIGURE CAPTIONS}

Figure 1. Motion of flat plate $\mathrm{AB}$ under the effect of aerodynamic forces $(F, N)$ and weight $(m g)$, gliding at constant angle of attack $i$, according to Zhukowsky [16].

Figure 2. Center of gravity trajectories as obtained from Eq. 6. The variety of solutions depends upon inertia and aerodynamic conditions through parameter A. The legends are Zhukowsky's originals [16]. 
Figure 3. Idealized flowfield around a rectangular flat plate falling while rotating in calm air, according to Zhukowsky [24].

Figure 4. Undulating trajectory of a hand glider's center of gravity, as suggested by Lanchester [7, 17].

Figure 5. Center of gravity trajectories as obtained by Lanchester through Eq. 28 [17].

Semicircular trajectories, numbered 4 in the picture, represent the stability limit (see similarity with Fig.2).

Figure 6. Sketch of flat plate gliding at angle $\gamma$, showing the speed V and angle of attack $\alpha$ in Painlevé's stability analysis [29].

Figure 7. Sketch of complex, three-lifting-surface aircraft (canard elevator, wing and fixed horizontal stabilizer) used by Bothezat in his six degrees of freedom stability studies [32].

Figure 8. Different types of perturbation damping shown in Bothezat's Thesis [32].

Figure 9. Sketch of airplane, with wing (S1 in the picture) and horizontal tailplane (S2), as idealized by Bryan in his stability studies [15]. 